Please do not remove this page

RMIT

UNIVERSITY

\title{
Steady state distribution of a hyperbolic digital tanlock loop with extended pull-in range for frequency synchronization in high doppler environment
}

Sithamparanathan, Kandeepan

https://researchrepository.rmit.edu.au/esploro/outputs/9921857834601341/filesAndLinks?institution=61RMIT_INST\&index=null

Sithamparanathan, K. (2009). Steady state distribution of a hyperbolic digital tanlock loop with extended pull-in range for frequency synchronization in high doppler environment. IEEE Transactions on Wireless Communications, 8(2), 890-897. https://doi.org/10.1109/TWC.2009.071337

Document Version: Accepted Manuscript

Published Version: https://doi.org/10.1109/TWC.2009.071337

Repository homepage: https://researchrepository.rmit.edu.au

(C) 2009 IEEE

Downloaded On 2023/04/26 10:03:17 +1000 
Thank you for downloading this document from the RMIT Research Repository.

The RMIT Research Repository is an open access database showcasing the research outputs of RMIT University researchers.

RMIT Research Repository: http://researchbank.rmit.edu.au/

\section{Citation:}

Steady state distribution of a hyperbolic digital tanlock loop with extended pull-in range for frequency synchronization in high doppler environment

See this record in the RMIT Research Repository at:

http://researchbank.rmit.edu.au/view/rmit:14471

Version: Accepted Manuscript

Copyright Statement: (c) 2009 IEEE

Link to Published Version:

http://dx.doi.org/10.1109/TWC.2009.071337

\section{PLEASE DO NOT REMOVE THIS PAGE}




\title{
Steady State Distribution of a Hyperbolic Digital TanLock Loop with Extended Pull-in Range for Frequency Synchronization in High Doppler Environment
}

\author{
Kandeepan Sithamparanathan, Member, IEEE,
}

\begin{abstract}
A hyperbolic arctan based Digital Tanlock Loop (D-TLL) operating with complex signals at base-band or intermediate frequencies in high Doppler environments is treated here. The arctan based loop, known as the tanlock loop (TLL), is used in software defined radio architectures for frequency acquisition and tracking. The hyperbolic nonlinearity intentionally introduced within the phase detector extends the pull-in range of the frequency for a given loop, compared to the normal D-TLL, allowing a wider frequency acquisition range which is suitable for high Doppler communications environment. In this paper we study the steady state phase noise performances of such a feedback loop for additive Gaussian noise using stochastic analysis. The stochastic model of a first-order hyperbolic loop and the theoretical analysis for the corresponding statistical distribution of the closed loop steady state phase noise are presented. The theoretical results are also verified by simulations.
\end{abstract}

\section{Index Terms}

Digital Tanlock Loop, D-TLL, arctan, hyperbolic loop, steady state distribution, steady state phase noise, pull-in range, Frequency Synchronization

\section{INTRODUCTION}

Synchronization of carrier frequency and phase is a well know problem in the field of communications [26]-[35], in this paper we address the phase synchronization problem using the tan locked loops. Analog and Digital, Tanlock Loops (TLL) are extensively used in many applications such as

Manuscript submitted on December 14, 2007 to the IEEE Transaction on Wireless Communications, revised and submitted on April 3, 2008

Dr. Kandeepan is with the National ICT Australia and the Research School of Information Science and Engineering at the Australian National University. National ICT Australia is funded through the Australian Government's Backing Australia's Ability initiative, in part through the Australian Research Council. 
communications, radar, sonar, navigation etc, for controlling and tracking signals. In this paper we consider the usage of such loops for frequency synchronization in software implemented digital receivers. The treatment and noise analysis of the loops for such applications with the arctan based phase detector [1]-[8] (TLL) and the sinusoidal based phase detector (traditionally known as the phase-locked loop) [12]-[24] have been treated well in literature. The major difference between the two is that, the 'sine' based loop shows different loop characteristics to the 'arctan' based loop due to their individual functional behaviors. The 'arctan' based TLL is known to have extended tracking capabilities in general compared to the 'sine' based loops [1],[16]. The noise analysis for the 'sine' based loop is well treated in literature, namely [13]-[21], and likewise the noise analysis of the TLL is also well treated in [1]-[2]. In the recent years, variations to the traditional TLL by incorporating an additional delay in the loop (time delay tanlock loop - TDTL) is also investigated in [3], [4]. In this paper, we are interested in improving the acquisition performance of the TLL by considering an additional nonlinear signal processing block placed within the loop. The model we consider here is a hyperbolic nonlinear model together with an arctan function [10]-[11], which is different from the above mentioned 'arctan' only and 'sine' only models. Other nonlinear loop models [9] such as the 'logarithmic' model is also presented in [8] for TLL. From the models presented in [9], the hyperbolic model shows improved pull-in range [10]-[11] by trading off with the phase noise performances, hence our interest in this model, and we investigate the loop further in this paper. For applications with unknown frequency variation, with a high dynamic range caused due to heavy Doppler in the received signal, increased acquisition-range is quite important for accurate frequency synchronization. The hyperbolic loop may be used in such situations with careful considerations of the steady state phase noise which we present in this paper. Here we study the steady state statistical distribution of the hyperbolic loop and compare and contrast the phase noise performances with the normal D-TLL and the 'sine' phase detector based phase locked loop in the simulations for additive Gaussian noise. This would allow system engineers to understand the degradation in the phase noise performances of the hyperbolic loop and decide whether it is acceptable to deploy such loops in their systems. The stochastic model 
that we develop here for a first-order hyperbolic loop results in a first-order Markov process, and by using this Markov model we seek solutions for the steady state statistical distribution for the phase noise within the loop. The solution for the phase noise distribution involves in computing a hard to solve integral for which we adopt numerical techniques to perform the computation.

In Section-II we present the complex signal model that we consider here, the hyperbolic nonlinearity based TLL and its corresponding loop design. In Section-III we analyse the statistical properties of the hyperbolic phase detector when it operates under additive white Gaussian noise. In Section-IV we derive the first-order Markov process corresponding to the first-order loop presented in section-II. The calculation of the steady state distribution and the corresponding simulation results are presented in Sections-V and VI respectively. Sections-VII and VIII present the steady state phase jitter performance of the hyperbolic loop and the extended pull-in characteristics, respectively, and finally in section-IX we present two classical synchronization examples where the hyperbolic loop can be used for frequency synchronization and its advantages in real life.

\section{LOOP MODEL}

The generalised loop model of a typical Digital-TLL has an error detector (phase detector), a loop filter and a numerically controlled oscillator known as the NCO. The NCO is the equivalent of the voltage controlled oscillator (VCO) in its analog counterpart. In our model, the phase detector is a four quadrant $\arctan ($.$) function that maps the input argument onto the four quadrant$ phase plane followed by a hyperbolic function $\mathrm{g}($.$) . For a standard D-TLL the \mathrm{g}($.$) function is only$ a gain factor. A detailed block diagram of our loop design is depicted in Figure-1. In Figure-1 the thicker arrow lines denote complex signals (real and imaginary) and the thinner arrow lines denote real signals. Further, $\mathrm{D}(\mathrm{z})$ and $\mathrm{V}(\mathrm{z})$ are the transfer functions of the loop filter and the NCO, respectively.

The discrete complex single-tone signal $r$ input to the loop with frequency $f$, amplitude $A$, and an arbitrary phase shift of $\beta$, corrupted with additive white Gaussian noise (AWGN), is given by, 


$$
r[n]=A \exp \left\{2 \pi j f T_{s} n+j \beta\right\}+\eta[n]
$$

For the signal model we assume a line of sight scenario with a slowly fading (constant) amplitude A and a slowly varying (constant) phase $\beta$, which are true for an equalized channel in a slow flat fading environment. In (1), $T_{s}$ is the sampling period of the discrete signal, with $f_{s}=1 / T_{s}$ being the sampling frequency, and $\eta$ is the complex Gaussian noise process. The Gaussian noise is expressed by its in-phase and quadrature components as,

$$
\eta=n_{c}+j n_{s}
$$

where, $n_{c}$ and $n_{s}$ are two discrete independent Gaussian random processes with zero mean and $\sigma^{2}$ variance with a double sided power spectral density of $N_{0}$ (Watts/Hz) each. At this point we define the signal to noise ratio as $S N R=A^{2} / \sigma^{2}$. The output signal $x$ from the NCO is given by,

$$
x[n]=\exp (-j \theta[n])
$$

where, $\theta[n]$ is the $n^{t h}$ sample from the NCO. The received signal $r$ is multplied by $x$ using a complex multiplier to generate an error signal $e$. The output of the complex multiplier $e$ is then input to the arctan function to estimate the phase difference between the received and the local signals, that is,

$$
\varphi_{e}=\arctan (\operatorname{Im}\{e\} / \operatorname{Re}\{e\})
$$

where, $\operatorname{Im}\{$.$\} and \operatorname{Re}\{$.$\} are the imaginary and real parts of the complex signal respectively.$ The estimated phase error $\varphi_{e}$ is then input to the hyperbolic function $\mathrm{g}\left(\right.$.) to generate $\varphi_{h}$,

$$
\varphi_{h}=\sinh \left(\varphi_{e}\right)
$$

where, $g()=.\sinh ($.$) . We define \phi[n]$ as the actual phase difference between the local and the received signals, which is given by,

$$
\phi[n]=\theta_{i n}[n]-\theta[n]
$$


where, $\theta_{\text {in }}$ is given by,

$$
\theta_{i n}[n]=2 \pi f T_{s} n+\beta
$$

We also present here the linear model of the D-TLL in order to compare the hyperbolic loop. In the absence of noise, the output of the arctan function gives a precise estimate of the phase difference between $-\pi$ and $\pi$ (that is $\varphi_{e}=\phi$ ), this is also valid for very little noise, or very high $S N R$. In the ideal case (in the absence of noise) a linear model is sufficient to precisely define the D-TLL. Such a linear model is depicted in Figure-2. The corresponding closed loop transfer function $\mathrm{H}(\mathrm{z})$ defining the linear loop (in Figure-2), is given by,

$$
H(z)=\frac{D(z) V(z)}{1+D(z) V(z)}
$$

We note here that the linear model of the 'sine' phase detector based loop is also similar to (8) [16], [13]. Since we are interested only in a first-order loop the loop filter is considered to be of unity gain. The NCO transfer function $\mathrm{V}(\mathrm{z})$ is given by,

$$
V(z)=\frac{k}{z-1}
$$

where, $k$ is the NCO parameter that controls the loop, or equivalently considered as the closed loop gain of the first-order loop. Finally, we define the term closed loop bandwidth for the linear loop, as [13],

$$
2 B_{L}=\frac{B_{i}}{2 \pi j} \oint_{|z|=1} H(z) H^{*}\left(1 / z^{*}\right) z^{-1} d z
$$

where, $B_{i}$ is the noise equivalent bandwidth of the input signal. The loop bandwidth is used to conveniently define the steady state phase jitter performance of the linear loop, we present this here in order to compare and contrast the analytical results of the hyperbolic loop model given in Figure-1 with the linear model given in Figure-2. A table of solutions for the contour integral in (10) can be found in [13] for different orders of loop. 


\section{Phase Detector Characteristics}

The statistical properties of the hyperbolic arctan based phase detector are important to derive a stochastic model for the nonlinear loop considered here. In this section we present the statistical properties of the hyperbolic phase detector. Let us define $\alpha$ as,

$$
\alpha=\sinh (\arctan \{\operatorname{Im}\{\kappa\} / \operatorname{Re}\{\kappa\}\})
$$

where, $\kappa=r,(r$ is given by (1)) with $f=0$. It should be noted here that the characteristics of $\alpha$ is different from $\varphi_{h}$ (given by equation (4)), where the former is an open loop estimate and the latter is a closed loop estimate of the parameter of interest. Then from [10], [11], the statistical distribution $f_{\alpha}\left(\alpha ; \mu_{1}, \mu_{2}\right)$ of $\alpha$ is given by,

$$
f_{\alpha}\left(\alpha ; \mu_{1}, \mu_{2}\right)=\cosh (\alpha) \Gamma_{\alpha}(\alpha)
$$

where, $\Gamma_{\alpha}(\alpha), \mu_{1}$ and $\mu_{2}$ are given in (13), (14) and (15). (Please note the typo error in [10] for the expression $\Lambda$. Expression (14) is the corrected one)

$$
\Gamma_{\alpha}(\alpha)= \begin{cases}\exp (-\varsigma / 2)\left[\frac{1}{2 \pi}-\exp \left(\frac{\Lambda_{1}}{2}\right)\left(\frac{\Lambda_{1}}{2 \pi}\right)^{\frac{1}{2}} Q\left(\sqrt{\Lambda_{1}}\right)\right] & \text { for } 0.7325 \pi<\alpha \leq 3.6761 \pi \\ \exp (-\varsigma / 2)\left[\frac{1}{2 \pi}+\exp \left(\frac{\Lambda}{2}\right)\left(\frac{\Lambda}{2 \pi}\right)^{\frac{1}{2}}(1-Q(\sqrt{\Lambda}))\right] & \text { for }-0.7325 \pi<\alpha \leq 0.7325 \pi \\ \exp (-\varsigma / 2)\left[\frac{1}{2 \pi}-\exp \left(\frac{\Lambda_{2}}{2}\right)\left(\frac{\Lambda_{2}}{2 \pi}\right)^{\frac{1}{2}} Q\left(\sqrt{\Lambda_{2}}\right)\right] & \text { for }-3.6761 \pi<\alpha \leq-0.7325 \pi\end{cases}
$$

where,

$$
\begin{gathered}
\mu_{1}=A \sin (\beta), \mu_{2}=A \cos (\beta), \varsigma=\frac{\mu_{1}^{2}+\mu_{2}^{2}}{\sigma^{2}}=S N R, \Lambda=\frac{\left[\tan (\alpha) \mu_{1}+\mu_{2}\right]^{2}}{\left[1+\tan ^{2}(\alpha)\right] \sigma^{2}} \\
\Lambda_{1}=\frac{\left[\tan (\pi+\alpha) \mu_{1}+\mu_{2}\right]^{2}}{\left[1+\tan ^{2}(\pi+\alpha)\right] \sigma_{2}^{2}} \Lambda_{2}=\frac{\left[\tan (-\pi+\alpha) \mu_{1}+\mu_{2}\right]^{2}}{\left[1+\tan ^{2}(-\pi+\alpha)\right] \sigma^{2}} \text { and }, Q(x)=\frac{1}{2 \pi} \int_{x}^{\infty} \exp \left(-u^{2} / 2\right) d u
\end{gathered}
$$

Now, let us rewrite $\alpha$ as, 


$$
\alpha=\alpha_{m}+\nu
$$

where, $\alpha_{m}$ is the expected value of the random variable $\alpha$, given by,

$$
\alpha_{m}=\int_{-\pi_{0}}^{\pi_{0}} \alpha f_{\alpha}(\alpha) d \alpha
$$

with $\pi_{0}=3.6716 \pi$, and $\nu$ is a time varying process. The random process $\nu$ has similar statistical properties as $\alpha$ but with $\mu_{1}=0$ and $\mu_{2}=1$ (where, $\mu_{1}$ and $\mu_{2}$ are defined in (14)). That is, $\nu$ is a zero mean random process with the statistical distribution given by $f_{\alpha}(\alpha ; 0,1)$. Figure- 3 shows the hyperbolic open loop phase noise distribution for various $S N R$ levels. The distribution of the hyperbolic phase noise, similar to any other noise models, becomes wider as the $S N R$ reduces. However, when the mean input phase is changed, while the $S N R$ is a constant, the distribution shows some significant variations. Figure-4 shows the variation in the distribution when the input phase is changed. For higher input phase values (the input phase is $\beta$ in this case) the distribution becomes wider as expected, and this can be verified by considering the characteristics of the hyperbolic function given by (5).

Further, the combined (arctan and hyperbolic) phase detector gives a mean output error when additive noise is present in the input signal. That is $\alpha_{m} \neq \sinh (\beta)$, rather, it has a non-unity d.c. gain associated with it, especially when the $S N R$ is low. By considering the d.c. gain of the hyperbolic phase detector [10], [11] we can write the expected value of the output phase $\alpha_{m}$ as,

$$
\alpha_{m}=K_{p} \sinh (\beta)
$$

where, $K_{p}$ is the phase detector gain, given by,

$$
K_{p}=\left.\left(\frac{d \alpha_{m}}{d \alpha}\right)\right|_{\alpha=0}
$$

It should be noted here that $K_{p}$ is a function of the input $S N R$, and $K_{p}=1$ for $S N R>18 d B$ [10]. Now, we can re-write $\alpha$ as,

$$
\alpha=K_{p} \sinh (\beta)+\nu
$$


The variation of $K_{p}$ and the characteristic curves with $S N R$ for the hyperbolic phase detector can be found in [10], [11]. In the following section, the results in (20) is directly applied to the error signal $e$ at the output of the complex multiplier in order to derive the stochastic model for the hyperbolic loop.

\section{Stochastic Modeling}

In this section we provide a stochastic model for the first-order feedback loop given in Figure-1 with $\mathrm{D}(\mathrm{z})=1$. The error signal $e$ at the output of the multiplier is given by,

$$
e[n]=A \exp \{j \phi\}+\tilde{\eta}[n]
$$

where,

$$
\tilde{\eta}=\exp \{-j \theta[n]\} \eta[n]
$$

It is rather straight forward to verify that $\tilde{\eta}[n]$ has similar statistical properties as the original noise process $\eta[n]$ given by (2). i.e. $\tilde{\eta}[n] \sim C N\left(0, \sigma^{2}\right)$, where $C N(a, b)$ denotes a complex Gaussian distribution with mean $a$ and variance $b$. Then, by using equations (5) and (20), we can rewrite the process $\varphi_{h}[n]$ at the output of the hyperbolic phase detector as,

$$
\varphi_{h}=K_{p} \sinh (\phi[n])+\chi[n]
$$

where, $\chi[n]$ is statistically similar to $\nu$ described in equation (16). Then, by using the time domain expression for the NCO given by (9) and along with equations (5), (6) and (20), one could arrive at the following first-order stochastic difference equation for the phase error process $\phi[n]$ as,

$$
\phi[n+1]=\phi[n]-K_{p} k \sinh (\phi[n])+\Omega+k \chi[n]
$$

where, $\Omega=2 \pi f T_{s}$. Further, the steady state phase error $\varphi_{s s}$, the value of $\varphi_{e}[n]$ as $n \rightarrow \infty$, can be derived from (24) by letting $\varphi_{e}[n]=\varphi_{e}[n-1]=\varphi_{s s}$ for $S N R=\infty$. Thus, we get,

$$
\varphi_{s s}=\sinh ^{-1}(\Omega / k)
$$


The corresponding linear loop given in Figure-2 has a steady state phase offset of $\varphi_{s s-l}=\Omega / k$. As we see, the first-order loop has a non-zero steady state phase error, increasing with the received frequency $f$, imposing an upper limit on the maximum acquirable frequency for a frequency step input. This maximum acquirable frequency is known as the pull-in frequency $f_{\text {pull-in }}$ and is given by letting the steady state phase error to $\varphi_{e}=\pi$. Thus for the hyperbolic loop we get a higher pull-in frequency than the linear loop, which is the main attraction of the hyperbolic loop. In other words, a specific pull-in range could be achieved by the hyperbolic loop with a smaller value of $\mathrm{k}$ compared to the D-TLL. In the following sections we analyse the steady state phase noise performance of the hyperbolic loop by computing the steady state distribution, and compare the phase jitter performances with the D-TLL.

\section{Steady State Closed loop Phase Error Distribution: First Order Loop}

The steady state statistical distribution of the phase error process (phase noise) is presented here for the first-order loop described in (24). We adopt similar techniques used by Aaron in solving for the steady state distribution for a non-uniformly sampled digital phase-locked loop [12] that has a sinusoidal type of phase detector characteristics. From (24) we clearly see that $\phi[n]$ is a discrete time continuous variable Markov process of first order. The statistical distribution of such a process satisfies the Chapman-Kolmogrov equation [25] for a given initial phase error of $\phi[0]=\phi_{0}$, which is given by

$$
P_{n+1}\left(\phi \mid \phi_{0}\right)=\int_{-\pi_{0}}^{\pi_{0}} Q_{n}(\phi \mid z) P_{n}\left(z \mid \phi_{0}\right) d z
$$

where,

$$
P_{n}\left(z \mid \phi_{0}\right)=\sum_{m=-\infty}^{\infty} p_{n}\left(z+2 m \pi_{0} \mid \phi_{0}\right)
$$

Since $\phi[n]$ and $\chi[n]$ are independent (from (24)), the transition probability follows the distribution,

$$
Q_{n}(\phi \mid z)=\frac{1}{k} \sum_{m=-\infty}^{\infty} f_{\alpha}\left(\frac{\phi}{k}+2 m \pi_{0} ; A \sin (u), A \cos (u)\right)
$$


where, $u$ is given by,

$$
u=z-K_{p} k \sinh (z)+\Omega
$$

In (27), $p_{n}\left(\phi \mid \phi_{0}\right)$ is the distribution of $\phi[n]$ given the initial condition $\phi_{0}$, and in (28), $Q_{n-1}(\phi \mid z)$ is the distribution of the transition probability of $\phi[n]$ given that $\phi[n-1]=z$, which is periodic with a period of $2 \pi_{0}$. The integral in (26) is nontrivial to solve in order obtain a closed form solution, therefore we seek numerical techniques to solve the integral. Then, by using (26), and starting with $P_{0}\left(z \mid \phi_{0}\right)=\delta\left(z-\phi_{0}\right)$ for $\phi_{0}=0$, where $\delta(z)$ is the dirac-delta function, we iteratively compute the statistical distribution of the phase error process $\phi[n]$, by letting $\mathrm{n}=0,1,2$.. until the successive distributions for $\phi[n]$ shows negligible differences (i.e. distribution reaching the steady state). The values of $m$ for the summation in the integration process used here were $m=-4,3 \ldots 3,4$, and the required number of iterations to compute the steady state distribution from the C-K integral in (26) depends on the number of samples required for the loop to reach steady state, this again depends on the value of $\mathrm{k}$. For example, for $\mathrm{k}=1$ the loop theoretically achieves steady state with the first iteration, therefore the number of iterations to compute the steady state distribution from (26) is also one. In the following section we provide some simulation results to verify the theoretical analysis performed here.

\section{Simulation Results}

In this section we present the simulation results for the hyperbolic loop, and compare the simulated steady state distribution with the theoretically computed distribution from (26). Figure5 shows the simulated and the theoretical distributions for the phase error process. From the results we see the theoretical distribution match closely with the distribution obtained from simulations. In the figure, two cases with different $S N R, f$, and $k$ values are presented to verify our analytical model. Figure-6 depicts the steady state distribution for different values of $k$ for $S N R=7 d B$. Again, we see the simulated results match the theoretical results as shown in the figure. From Figure-6, we also see how the steady state distribution flattens out with increasing $k$ giving rise to higher phase noise within the loop. Therefore, it is quite important to maintain low values for 
$k$ to control the phase noise, but as we see from Section-VIII reducing $k$ theoretically reduces the pull-in range of the loop. However, by using the hyperbolic loop instead of the D-TLL one could reduce $k$ without reducing the required pull-in range of the loop, which is the main advantage of using the hyperbolic loop. We explain this further with a system example in Section-IX.

\section{Steady State Phase JitTer: First ORder Loop}

The steady state phase jitter (the square root of the phase error variance) is a key parameter describing the synchronization degradation in designing a communication system. Due to the random phase disturbances in the synchronized signal the probability of bit error in digital communications degrades and is usually quantified using the value of phase jitter. Here we present the steady state phase jitter of the hyperbolic loop and compare the performances with the D-TLL and the 'sine' phase detector based loop. The theoretical expression for the phase jitter of the linear loop is given by [13],

$$
\sigma_{\phi}=\sqrt{N_{0} B_{L}}
$$

where, $B_{L}=f_{s} k /(2-k)$. The phase jitter for the hyperbolic loop is computed by calculating the variance from the steady state distribution obtained from the $\mathrm{C}-\mathrm{K}$ integral. Figure-7 depicts the phase jitter performances of the hyperbolic loop. From the figure we see that the simulation results closely match with the theoretical results. Figure- 8 compares the phase jitter performance of the hyperbolic loop with the D-TLL and the 'sine' phase detector based loop. The degradation in the steady state jitter performances of the hyperbolic loop can be seen from this figure, especially for $S N R<13 d B$. This is the major drawback of the hyperbolic loop compared to the D-TLL. However, using the extended pull-in range property of the hyperbolic loop, the NCO constant $k$ can be reduced in order to achieve better jitter performances, we explain this in a later section as an example. 
VIII. EXTENDED PULL-IN FEATURES OF THE HyPERBOLIC D-TLL: NOISELESS ANALysis

In this section we analyse the extended pull-in capability of the hyperbolic loop for the noiseless case. For the D-TLL the pull-in range is found by letting $\phi_{s s-l}=\pi$ and obtaining an expression for $f$, resulting in,

$$
f_{\text {pull-in-DTLL }}=k f_{s} / 2
$$

Likewise, the theoretical pull-in range of the hyperbolic loop is obtained by letting $\phi_{s s}=\pi$, resulting in,

$$
f_{\text {pull-in-Hyp }}=\sinh (\pi) k f_{s} /(2 \pi)
$$

From (31) and (32), we see that the lock-in range of the hyperbolic loop is theoretically improved by a factor of 3.67 compared to the D-TLL. We show the corresponding results in Figure- 9 by tracking a $910 \mathrm{~Hz}$ signal with $k=0.1$ at $f_{s}=5 k H z$. The figure shows the error signal within the loop, which should reach a steady state (constant) value after acquiring the received frequency. The theoretical maximum frequency acquirable by the D-TLL for the given values of $k$ and $f_{s}$ is $250 \mathrm{~Hz}$ (from (31)), and as we see from Figure-9 the hyperbolic loop achieves lock for a frequency of $910 \mathrm{~Hz}$ (that is, the error signal of the hyperbolic loop becomes constant after acquiring the received frequency whilst the other does not), which is almost 3.6 times the acquirable frequency of the D-TLL, this verifies the claim of having extended pull-in range when using the hyperbolic loop over the D-TLL. The figure also shows the 'sine' based loop which is unable to acquire the frequency for the given case.

\section{A. Phase Plane Analysis of the Hyperbolic Loop}

The acquisition performance of the first-order nonlinear loop is best analysed by considering the phase plane trajectories which are the possible solutions for the difference equation described in (24) in the absence of noise. The phase plane portrait is generated by plotting the phase error on the $\mathrm{x}$-axis and the frequency error on the y-axis, with time. This shows us the convergence of the loop towards the equilibrium point corresponding to the steady state phase and the frequency error 
values. Here we show a few phase plane trajectories obtained using simulations for the noiseless case in order to understand the behavior of the nonlinear loop.

Figure-10 and Figure-11 show the phase plane trajectories for $k=1$ and $k=0.8$, respectively. The acquisition process of the signal frequency is well observed in the two figures with the frequency error eventually becoming zero and the loop attaining lock. The arrow markings shown in Figure-10 denote the directions of the trajectories starting at different frequency errors and converging towards a zero frequency error. The phase plane portrait presented in Figure-11 also follows similar directions as per the trajectories given in Figure-10. The differences in the acquisition processes between the D-TLL, 'sine' based loop, and the hyperbolic loop are clearly observed in Figure-11. From the figure, we see how the steady state phase error values for the hyperbolic loop are squeezed-in when compared with the D-TLL and the 'sine' based loop theoretically achieving a greater lock-in range. We also observe that the 'sine' loop diverges from the steady state point between $-\pi$ and $\pi$ which is an indication of the first order 'sine' loop being unable to acquire the frequency. For higher values of loop gain (higher values of $k$ ), the hyperbolic loop however tends to overshoot with higher values, and in such situations a second-order loop is required for convergence. Such analysis using control theories for nonlinear loops are subjected to further studies and is beyond the scope of this paper.

\section{Synchronization Systems Examples Using the Hyperbolic Loop}

In this section we provide two examples (two scenarios) to show how the hyperbolic loop outperforms the traditional D-TLL. In the first example, we fix the required pull-in range, or in other words we have a predefined value for the signal frequency pull-in range, and show how the steady state phase jitter could be reduced by reducing the loop gain using the hyperbolic loop. In the second example, we fix the steady state phase jitter to be a predefined value and improve the frequency pull-in range of the received signal using the hyperbolic loop. The former example is best suited for applications that require improved phase jitter performances (typically any mobile digital communication system), and the latter is best suited for receivers requiring extended pull-in 
range capabilities (typically highly mobile communication links such as low earth orbital satellite communications or wireless communications on high speed trains at higher frequency bands).

\section{A. Example-1:}

In this example, let us define a required pull-in range of $\pm 2 \times 10^{-3}$ percent of the carrier frequency $f_{c}$. For $f_{c}=2.4 G H z$, the lock-in frequency requirement is then given by $\pm 48 k \mathrm{~Hz}$. Considering the feedback loops to be used at base-band, after the down conversion process, to track residual frequency error present in the received signal with a sampling frequency of $f_{s}=4.8 \times 10^{5} \mathrm{~Hz}$, the minimum required value of $k$ for the D-TLL computed from equation (31) is given by $k=0.2$. For the same scenario however, the hyperbolic loop requires a minimum value of $k=0.0544$ to achieve the same pull-in range. Therefor, by reducing the NCO parameter $k$ we can reduce the phase jitter within the loop. The improvement on phase jitter by using the hyperbolic loop is depicted in Figure-12 for $k=0.2$ and $k=0.06$. From the figure, we clearly see the improvement on the phase jitter performances. The system shows an improvement of around $6 \mathrm{~dB}$ to $8 \mathrm{~dB}$ above the threshold and around $1 \mathrm{~dB}$ to $4 \mathrm{~dB}$ of improvement below the threshold. The SNR improvement becomes higher when the required pull-in range increases further.

\section{B. Example-2:}

In the second example, for a specific system operating at $S N R=15 \mathrm{~dB}$ let us define an acceptable phase jitter that the receiver could tolerate to be $\sigma_{\phi}=0.05 \mathrm{rad}$. For the D-TLL, using Figure-12 we see that a value of $k=0.2$ can achieve the required phase jitter performance. Using Figure-12 again, the same phase jitter performance also can be achieved using the hyperbolic loop for $k=0.2$. The maximum acquirable frequency offset (Doppler frequency) for the D-TLL in this case is given by $\pm 48 \mathrm{kHz}$, which corresponds to a maximum transmitter-receiver relative speed of $v=26000 \mathrm{kmhr}^{-1}$ in a mobile communication environment. This is a typical LEO satellite system operating at $\mathrm{Ku}$-band $(20 \mathrm{GHz})$. However, by using the hyperbolic loop, the maximum theoretical frequency offset (Doppler frequency) that could be achieved is $\pm 176 \mathrm{kHz}$, which then 
can be used with a Ka-band $(30 \mathrm{GHz})$ communication link for the same LEO satellite system without any degradation in the phase jitter performances.

\section{COnClusion}

A hyperbolic nonlinearity based digital tanlock loop and its corresponding steady state and acquisition performances were presented for a first-order loop. The hyperbolic loop gives an extended pull-in range at the expense of the steady state phase jitter for a given loop gain when compared with a digital tanlock loop. However, we have shown that, by using the hyperbolic loop we could achieve equivalent or better phase jitter performance for a specific system by effectively reducing the loop gain. This is made possible by extending the pull-in range when using the hyperbolic loop.

A stochastic difference equation for the hyperbolic loop was presented and the corresponding steady state closed loop distribution of the phase noise was obtained using the ChapmanKolmogrov equation. The theoretical results were verified using simulations. The steady state phase jitter of the hyperbolic loop was also analysed and was compared with the traditional digital tanlock loop and the 'sine' phase detector based phase locked loop. Finally, two classical examples were provided where the hyperbolic loop could improve the performances of the receiver in wireless communications. 


\section{REFERENCES}

[1] J.C.Lee and C.K.Un,’Performance Analysis of Digital Tanlock Loop,” IEEE Trans on Communications, vol. 30, No.10, pp. 2398-2411, October 1982.

[2] C.A.Pomalaza-Raez,’'Noise Analysis of a Digital Tanlock Loop,' IEEE Trans on Aerospace and Electronic System,vol. 24, pp. 713-718, 1988.

[3] Z. M. Hussain et. al., ”A time-delay digital tanlock loop," IEEE Transactions on Signal Processing, vol. 49, no. 8, pp. 1808-1815, Aug. 2001.

[4] Al-Qutayri, M.A.; Al-Araji, S.R.; Al-Moosa, N.I., 'Improved First-Order Time-Delay Tanlock Loop Architectures' IEEE Transactions on Circuits and Systems-I Volume 53, Issue 9, Sept. 2006 Page(s):1896 - 1908

[5] Makarious, A.H.; Farrell, P.G.; 'Novel p.s.k. tanlock loop' Electronics Letters, Volume 16, Issue 25, December 41980 Page(s):957 - 958

[6] Cho, W.D.; Un, C.K.; 'On improving the performance of a digital tanlock loop' Proceedings of the IEEE, Volume 75, Issue 4, April 1987 Page(s):520 - 522

[7] Hwa Kim; Chong Un; Jae Chon Lee; 'The N-Phase Digital Tanlock Loop for Tracking Suppressed-Carrier N-ary PSK Signals',IEEE Transactions on Communications, Volume 33, Issue 9, Sep 1985 Page(s):904 - 910

[8] S. Kandeepan and S. Reisenfeld, "Performance Analysis of a Logarithmic based Phase Detector for Tan-Locked Loops" IEEE 18th International Symposium on Personal, Indoor and Mobile Radio Communications, Athens, 2007 3-7 Sept. 2007.

[9] S. Kandeepan and S. Reisenfeld, "Phase detector models and their performances for IF/baseband frequency recovery for complex envelope based DSP implemented PLL” The 8th International Conference on Communication Systems,25-28 Nov 2002 Page, $774-778$

[10] S.Kandeepan, S.Reisenfeld,'Performance Analysis of a Digital Phase-Locked Loop with a Hyperbolic Nonlinearity," IEEE International Conference on Information, Communications and Signal Processing ICICS 20056-9 Dec, Bangkok, 2005.

[11] S Kandeepan, Synchronisation techniques for digital modems, PhD thesis, University of Technology, Sydney, 2003.

[12] A. Weinberg, Bede Liu, "Discrete Time Analyses of Nonuniform Sampling First- and Second-Order Digital Phase Lock Loops", IEEE Transactions on Communications, Vol. 22, No.2, pp 123-137, February 1974.

[13] W.C.Lindsey and C.M.Chie,”A Survey of DPLL” Proceedings of the IEEE pp296-317 April 1981.

[14] H. Meyer, G Ascheid, "Synchronisation in Digital Communications", vol-1, John Willey and Sons, 1990.

[15] A.J. Viterbi, ”Principles of Coherent Communications", McGraw-Hill, 1966.

[16] F.M.Gardner," Phase Lock Techniques,” NY, Willey, 1979.

[17] W. C. Lindsey, "Synchronization Systems in Communication and Control", Englewood Cliffs, NJ: Prentice Hall, 1972.

[18] W. C. Lindsey, M. K. Simon, ”Telecommunication Systems Engineering”, Englewood Cliffs, NJ Prentice Hall, 1973.

[19] S. C. Gupta, "Phase-Locked Loops, ” Proceedings of IEEE vol. 63, pp 291-306, Feb 1975.

[20] C. M. Chie, "Mathematical analogies between first-order digital and analog phase-locked loops," IEEE Transactions on Communications, Vol COM-26, pp 860-865, June 1978. 
[21] G. Skiller, D. Huang, ”The Stationary Phase Error Distribution of A Digital Phase-Locked Loop”, IEEE Transactions on Communications, Vol. 48, No.6, pp 925-927, June 2000.

[22] M. P. Fitz and R. J.-M. Cramer,"A Performance Analysis of a Digital PLL Based MPSK Demodulator," IEEE Trans on Communications, vol. 43 No.2/3/4, pp. 1192-1201, Feb/Mar/Apr 1995.

[23] J.V.Lorenzo-Ginori and J. A. Naranjo-Bouzas,"All-Digital PLL with Extended Tracking Capabilities," IEEE Electronics Letters, vol. 33 No.18, Aug 1997, pp. 1519-1521.

[24] R.C.Tausworthe,"A Second/Third-Order Hybrid Phase Locked receiver for Tracking Doppler Rates” JPL Tech Rep, 321526,vol.1,pp 42-45.

[25] J. L. Doob, Stochastic Process, New York, Wiley, 1953, pp196-198.

[26] S. Kandeepan, Omar. H, Qian, A Complex Signal Based Digital Phase-Locked Loop with arctan Phase Detector Implemented on High Speed FPGA, IEEE conf on Info Comm \& Signal Processing 2007, Singapore

[27] S. Kandeepan, A Software Phase Locked Loop from Theory to Practice: TMS320C6000 DSP Based Implementation and Analysis, AusWireless conf 2006, Sydney March 13-16.

[28] S. Kandeepan, S. Reisenfeld. Frequency Error Correction for OFDM based Multicarrier Systems and Performance Analysis, IEEE Int Conf on Information, Communications and Signal Processing ICICS, 6-9 Dec, Bangkok, 2005.

[29] S. Kandeepan, and S. Reisenfeld, Acquisition Performance of a Digital Phase Locked Loop with a Four-Quadrant arcane based Phase Detector, IEEE International Symposium on Intelligent Signal Processing and Communications, Nov 2004.

[30] S. Kandeepan, and S. Reisenfeld, Fast Doppler Tracking DSP-Based Earth Station Modem for LEO Satellite Applications, IEEE Int Symp on Intelligent Signal Processing and Communications, Nov 2004

[31] S. Kandeepan, and S. Reisenfeld, Performance Analysis of DPLL with statistical filters for carrier synchronisation, IEEE International Conference on Signal Processing and Communications, Dec 2004.

[32] S. Kandeepan and S. Reisenfeld, Performance Analysis of a Correlator Based Maximum Likelihood Frequency Estimator, IEEE International Conference on Signal Processing and Communications, Dec 2004

[33] S. Kandeepan. and S. Reisenfeld, "DSP Based Frequency Estimation Techniques and Their Relative Performances", Symp on Communication Systems, Networks and Digital Signal Processing (CSNDSP2002), July 15-17, Staffordshire.

[34] S. Kandeepan and S. Reisenfeld, "Frequency Jitter of a Digital Phase-Locked Loop and comparison with a Modified CRB", IEEE International Conference on Communication Systems 2002, Nov 25-29. Singapore.

[35] S. Kandeepan and S. Reisenfeld, "A Complex Envelope Based 2nd Order Digital Phase Locked Loop and its Performance", IEEE International Symposium on Intelligent Signal Processing and Communication Systems 2001(ISPACS 2001) Proceedings of, Nov 20-23, Nashville 


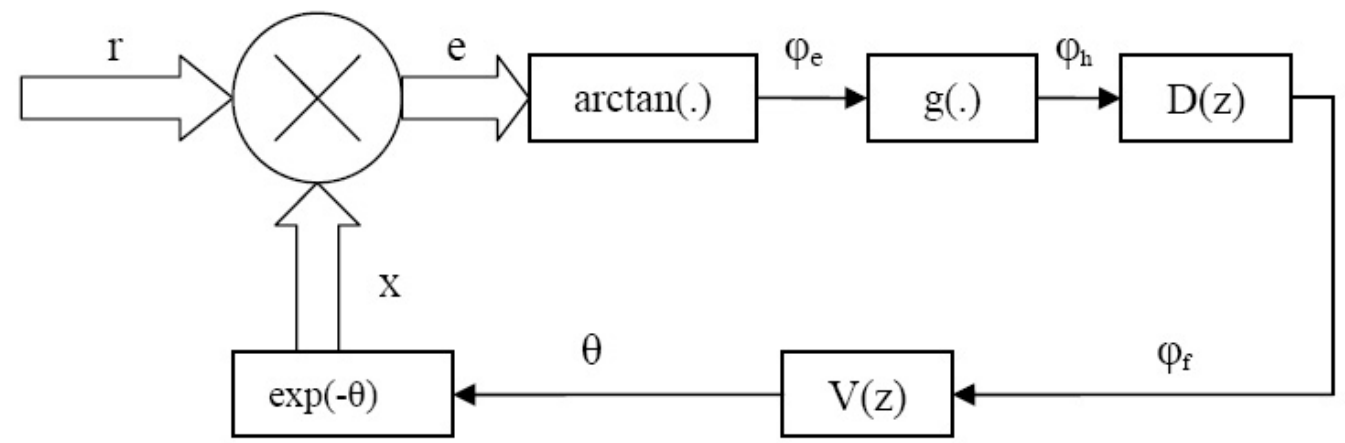

Fig. 1. Block Diagram of the complex signal based hyperbolic Digital Tanlock Loop

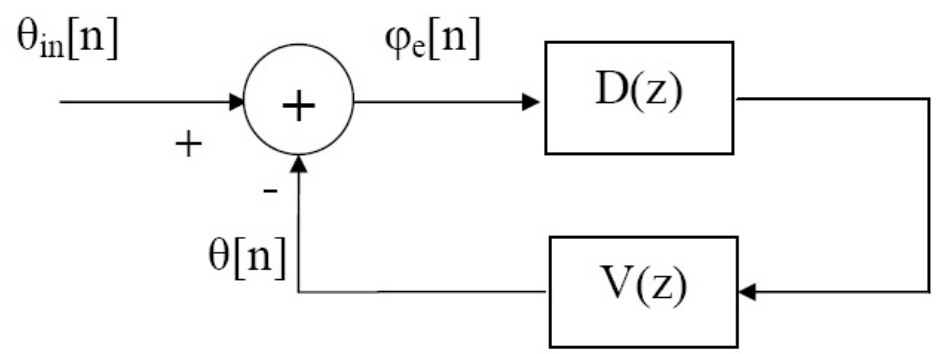

Fig. 2. Linear model of the D-TLL and the 'sine' phase detector based digital phase-locked loop in the absence of noise

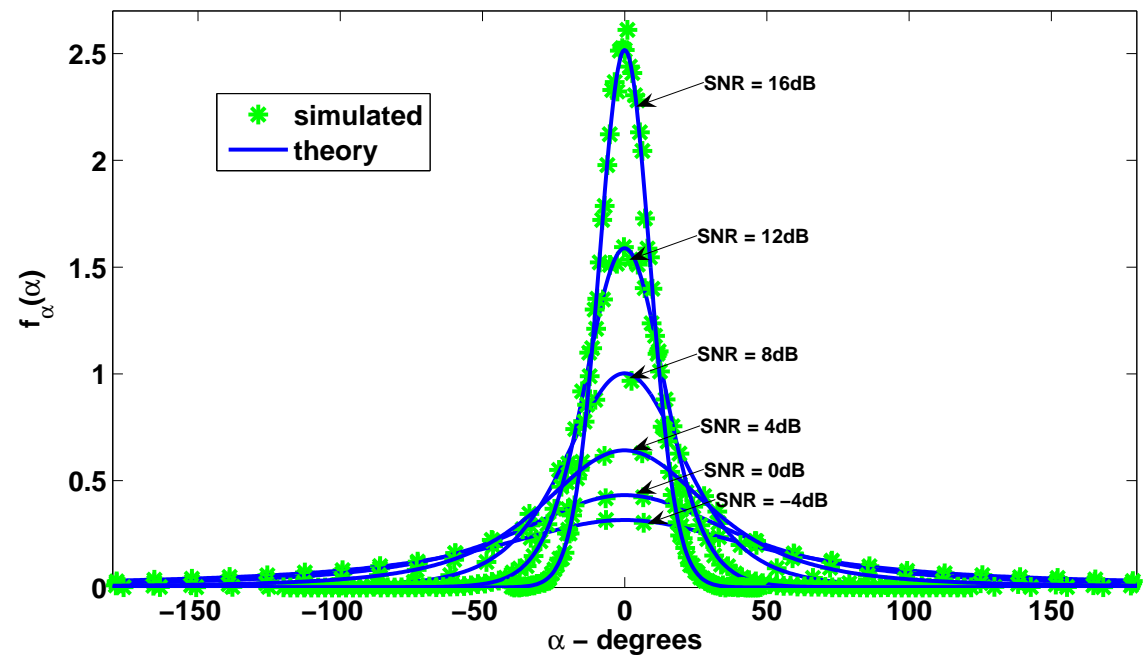

Fig. 3. Open loop hyperbolic phase noise distribution for various $S N R$, with $\beta=0$ 


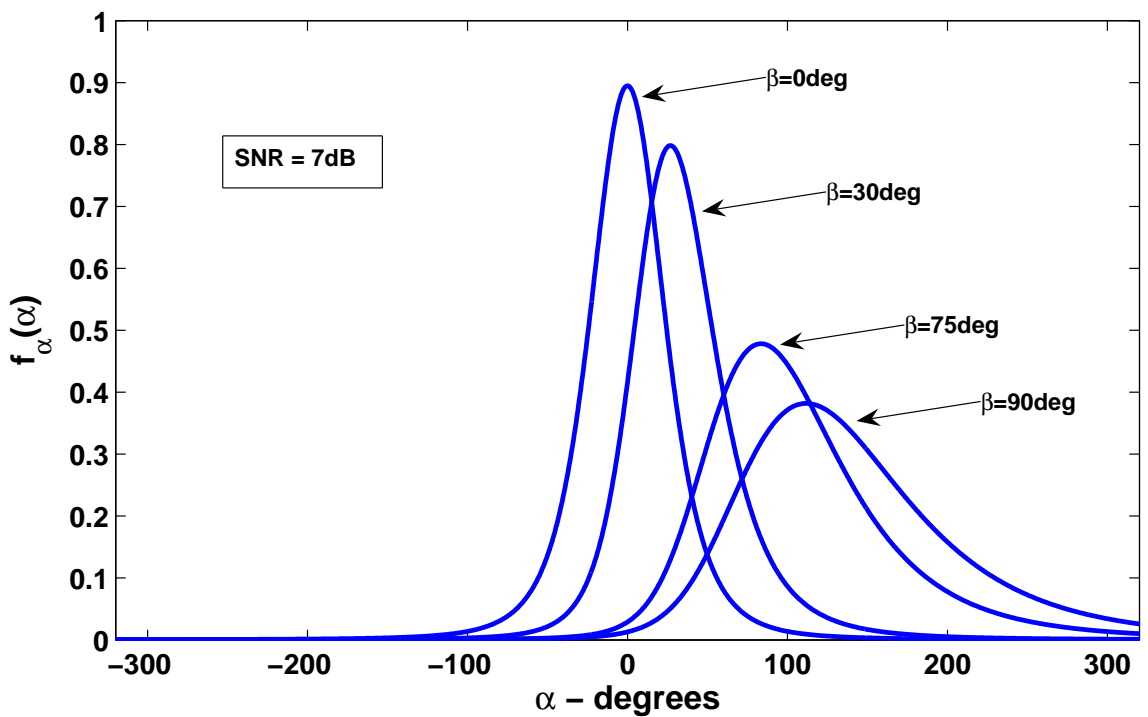

Fig. 4. Open loop hyperbolic phase noise distribution variation with $\beta$

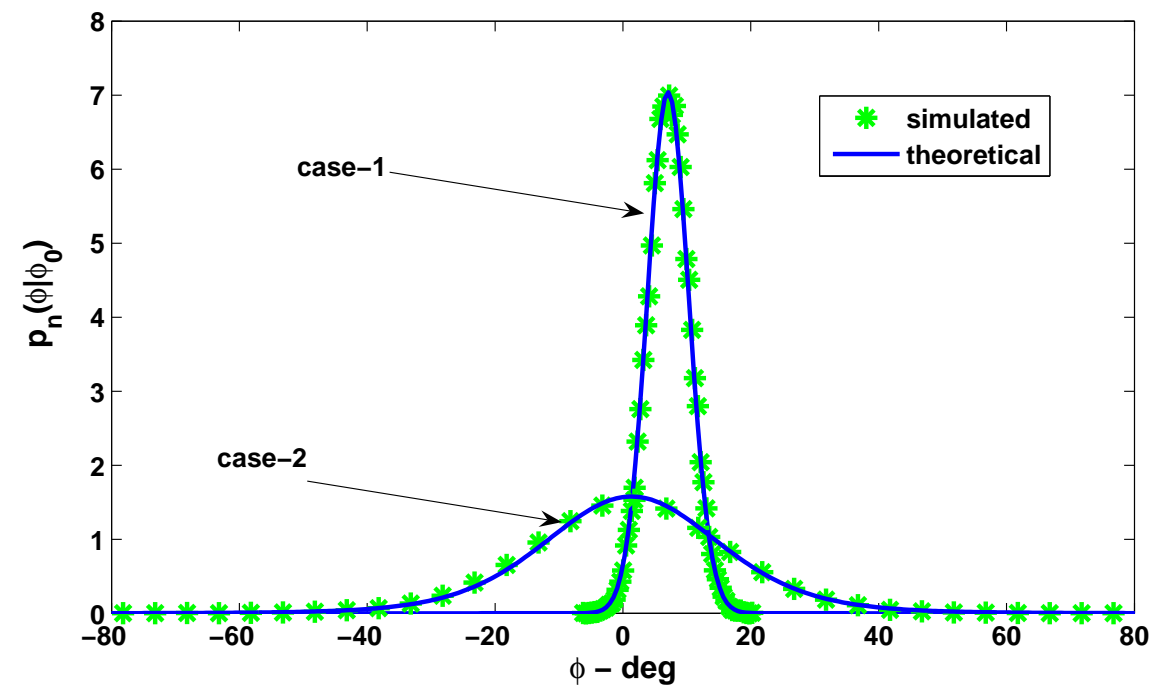

Fig. 5. Steady state phase error distributions for the first-order hyperbolic loop for, case-1: $S N R=25 d B, k=1, f=200 H z$, $f_{s}=10 \mathrm{kHz}$, case- $2: S N R=10 \mathrm{~dB}, k=0.8, f=20 \mathrm{~Hz}, f_{\mathrm{s}}=10 \mathrm{kHz}$ 


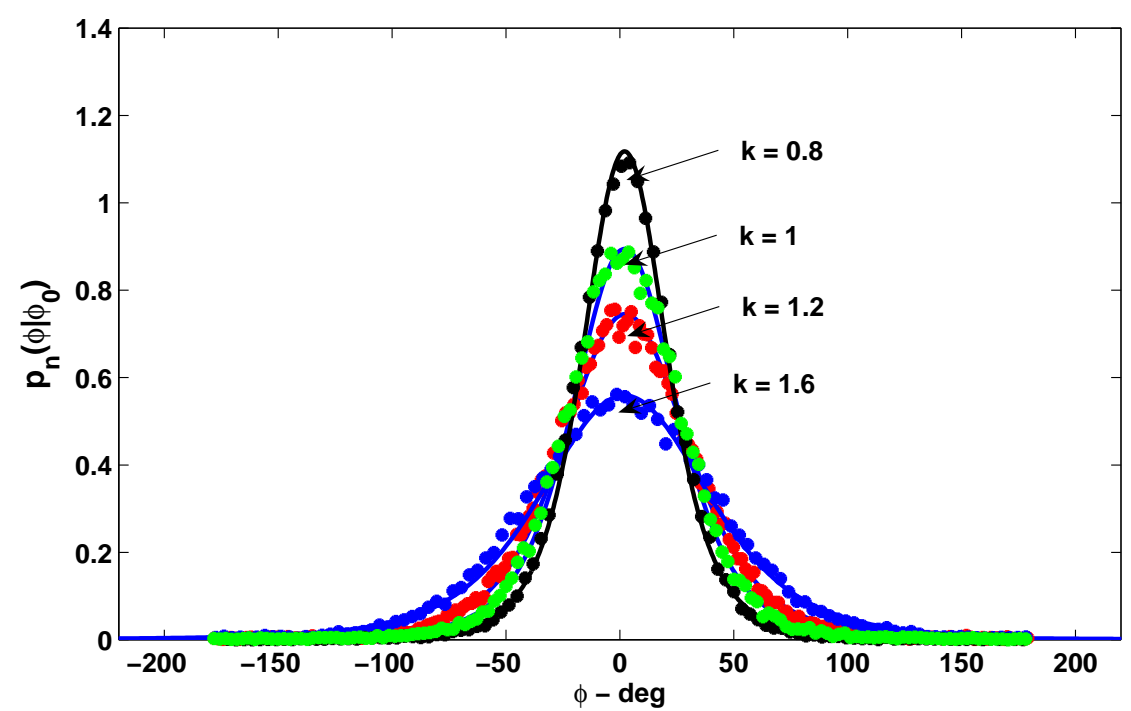

Fig. 6. Steady state phase error distribution for the first-order hyperbolic loop for $f=50 \mathrm{~Hz}, f_{s}=10 \mathrm{kHz}$ and $S N R=7 d B$; - Theory; * simulation

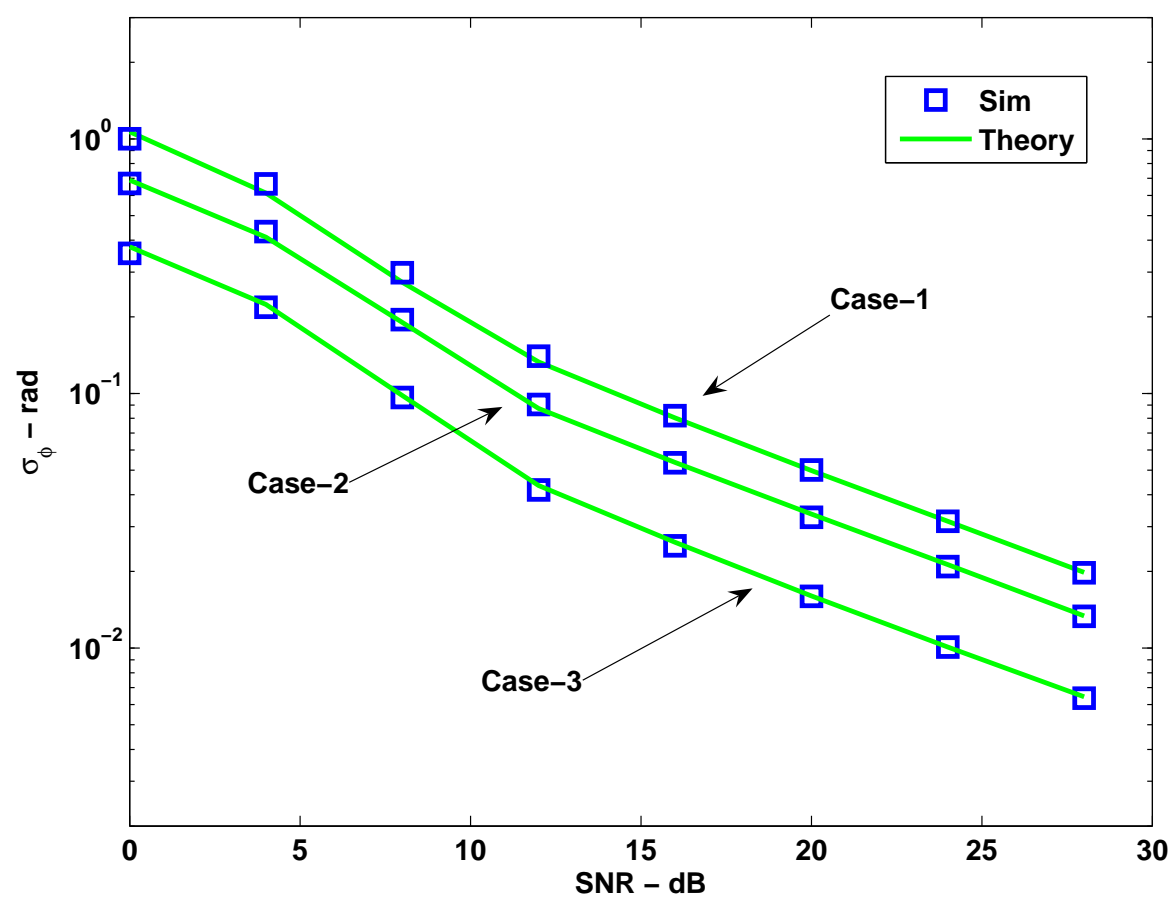

Fig. 7. Steady state phase jitter for the first-order hyperbolic loop; case-1: $k=0.6, f=5 H z, f_{s}=500 H z$, case-2: $k=0.2, f=$ $10 \mathrm{~Hz}, f_{s}=2000 \mathrm{~Hz}$, case- $3: k=0.04, f=5 \mathrm{~Hz}, f_{s}=5000 \mathrm{~Hz}$ 


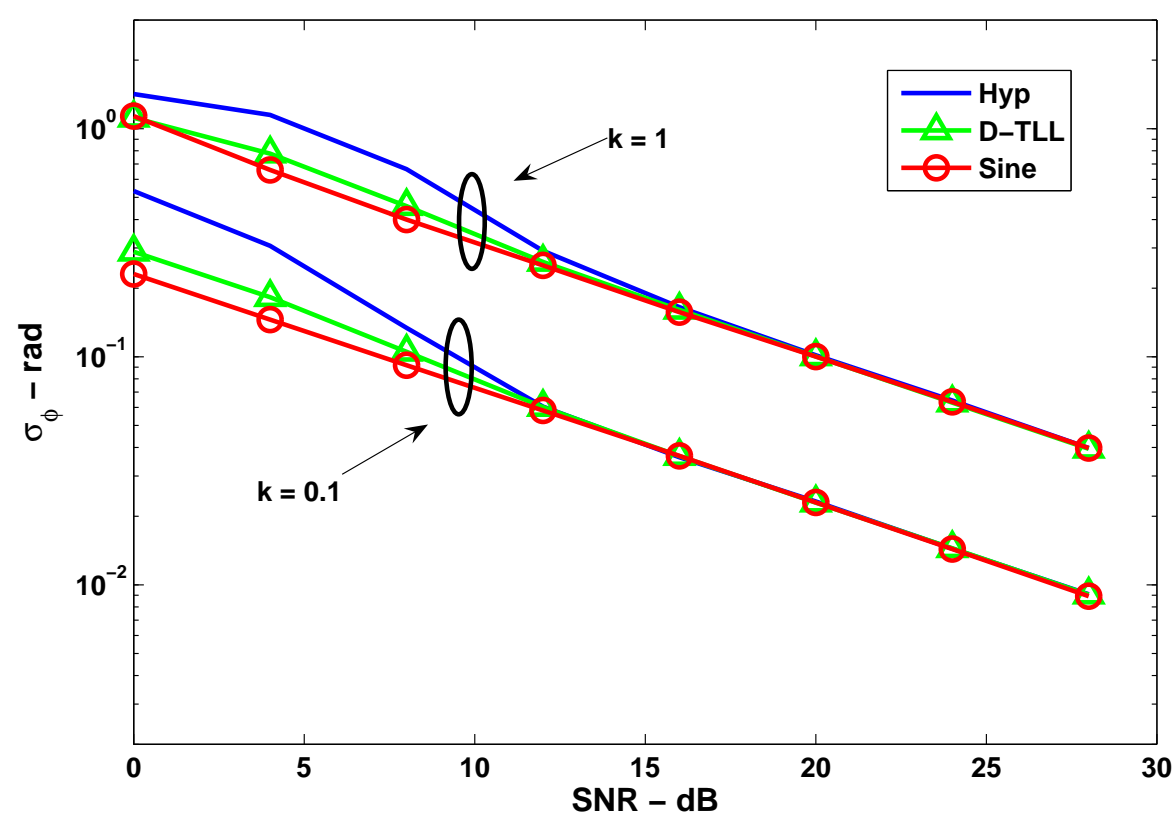

Fig. 8. Comparing the steady state phase jitter of the first-order hyperbolic loop with the Digital-TLL, and the 'sine' phase detector based PLL; $f=50 \mathrm{~Hz}, f_{s}=50 \mathrm{kHz}$

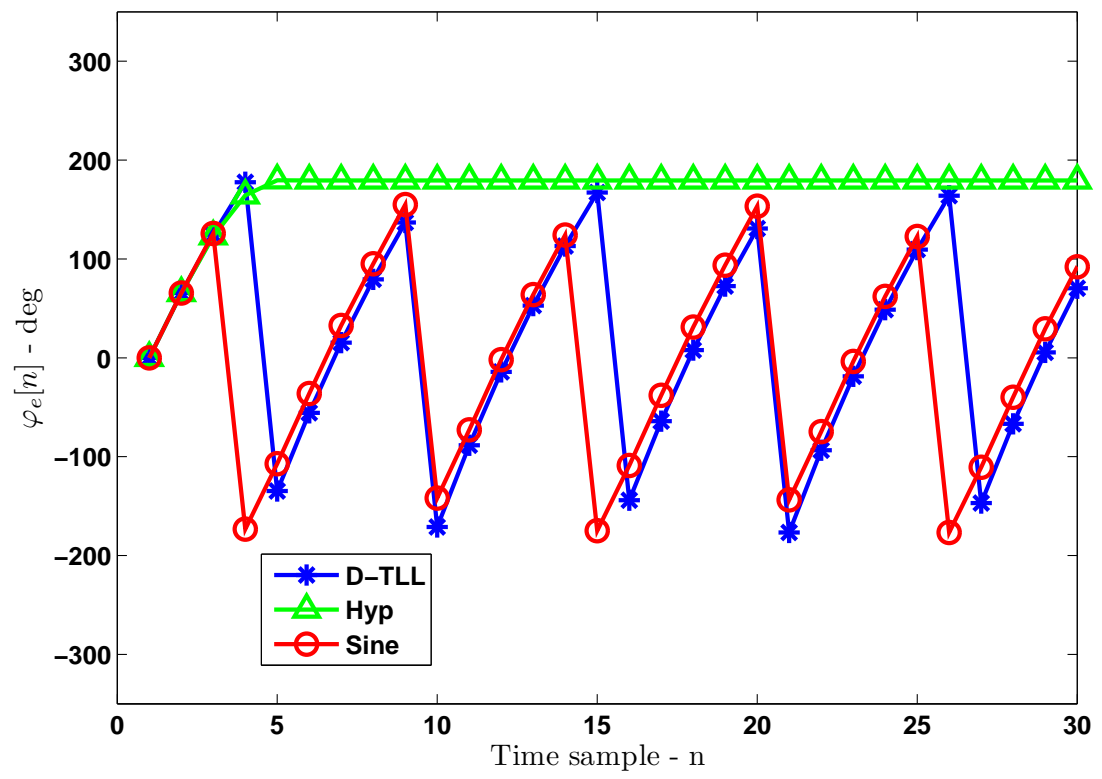

Fig. 9. Comparing the pull-in capability of the first-order hyperbolic loop with the Digital-TLL and the 'sine' based loop; $f=910 \mathrm{~Hz}, f_{s}=5 k H z$ and $k=1$ 


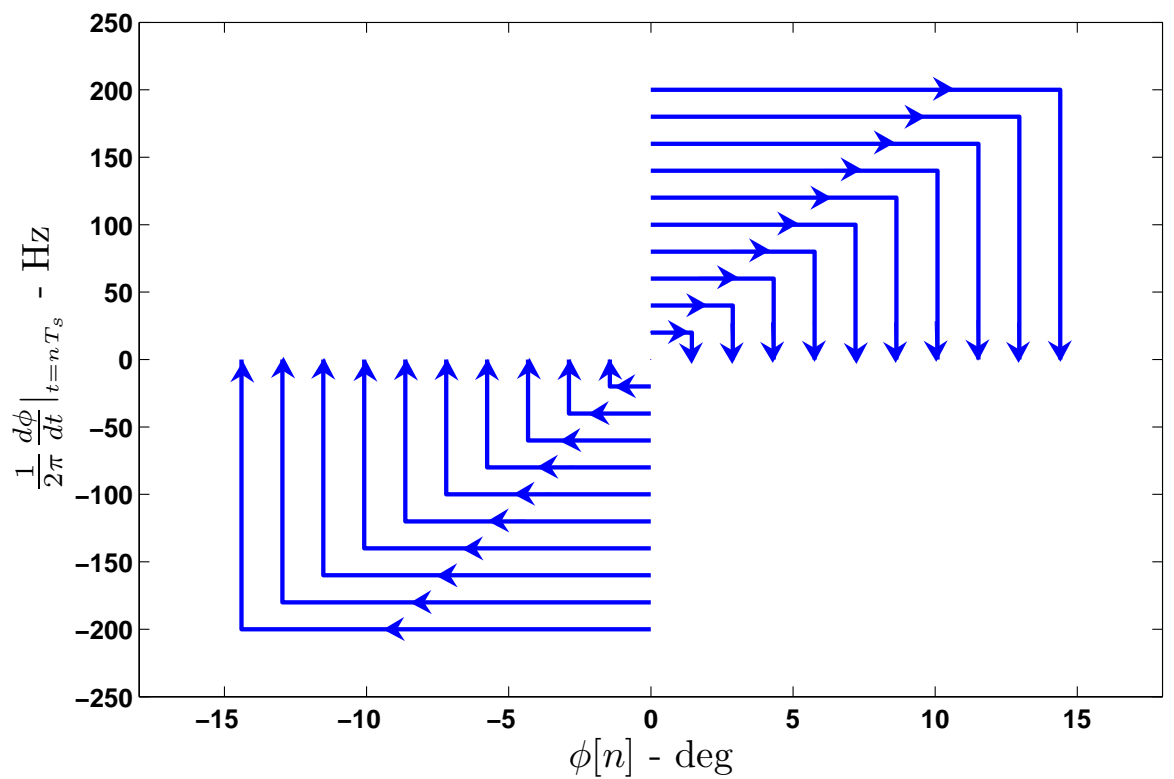

Fig. 10. Phase Plane Portrait: Hyperbolic loop with $f_{s}=5 k H z$ and $k=1$

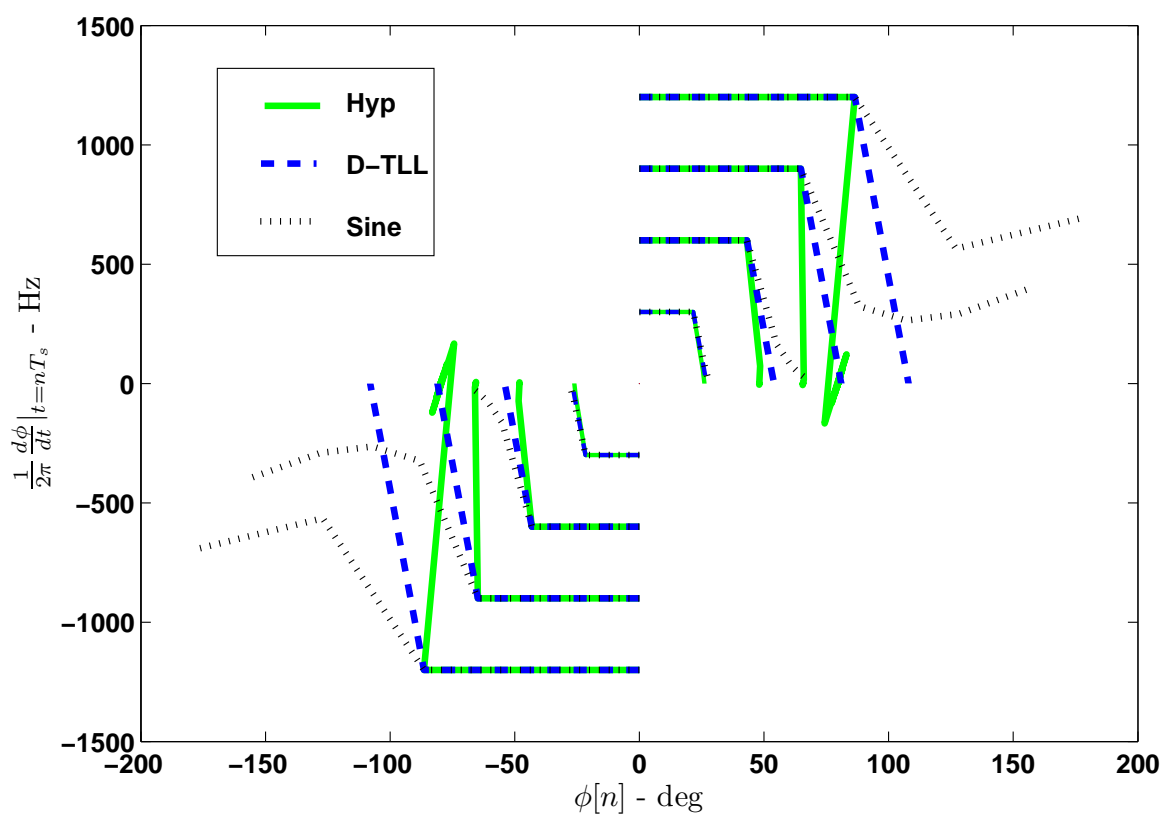

Fig. 11. Comparing the Phase Plane Portraits of the Hyperbolic, D-TLL, and the 'sine' phase detector based digital phase locked loop, with $f_{s}=5 \mathrm{kHz}$ and $k=0.8$ 


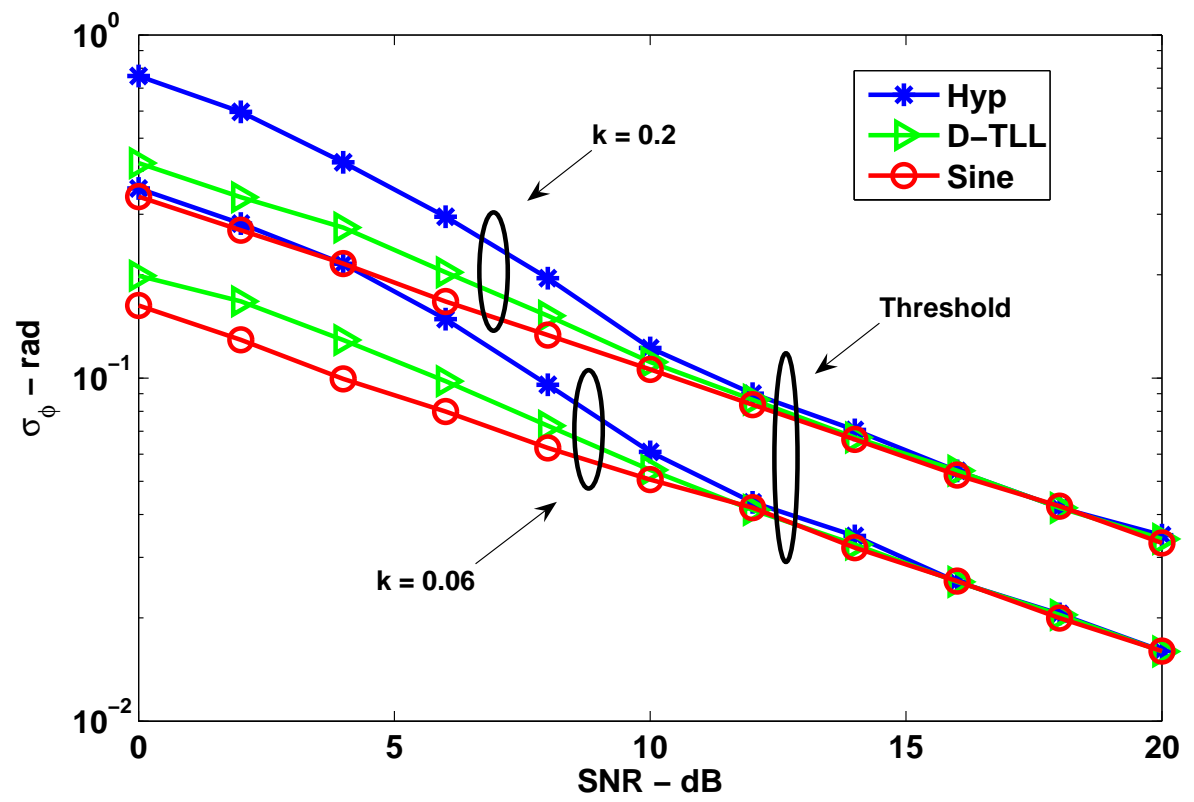

Fig. 12. Example-1: Performance improvement in steady state phase jitter using the hyperbolic loop, with $f_{s}=4.8 \times 10^{5}$ 